\title{
Applying Structured Scheduling to Increase Performance in Rural Demand-Response Transportation
}

\author{
Kai Monast and Joshua Worthy \\ North Carolina State University
}

\begin{abstract}
Many rural demand-response transportation systems have increased ridership to a level that the systems feel they need technology or increased scheduling and vehicle capacity. Instead of adding resources, capacity can be expanded and performance can be increased by applying a scheduling structure. The scheduling structure proposed in this research applies directly to systems that share specific geographic characteristics. For these areas, trips are assigned to runs based on time and location, which results in increased performance and vehicle utilization. The structure enables trips to be scheduled during the booking process using simple and easy-tounderstand rules that allow the customer to select the appropriate route.

This research explains how to establish structured rural demand-response transportation service and enumerates its benefits through a case study consisting of actual service data. The case study shows a reduction in service miles by 27 percent due to implementation of structured scheduling.
\end{abstract}

\section{Introduction}

Rural demand-response transportation (DRT) is inefficient to deliver, time-consuming to schedule, and requires a large vehicle fleet whose capacity tends to be underutilized. Many rural DRT systems have increased ridership to a level that has 
led to the perception that technology or increased scheduling and vehicle capacity are warranted to meet the current demand. The greatest opportunity for increasing rural DRT performance and decreasing the scheduling effort is for transportation systems to structure the service instead of continually adapting to the stated demand. Structured service means that the rural DRT has planned a route structure by area and time to provide understandable, consistent service that meets the needs of the customers.

This research applies most directly to rural demand-response transportation systems that possess specific characteristics, including one, and only one, urban area in or near the center of the service area with the basic medical, employment, and shopping destinations; major roads that lead to the urban area; and a density that is high enough to allow service to all destinations within a reasonable time.

There are numerous benefits to structured service. The structure groups trips to runs, which increases performance and vehicle utilization while decreasing the demand for more vehicles. Where a structure exists, the trip is scheduled during the reservation/booking process using simple and easy-to-understand rules that allow the customer to select the most appropriate route.

Scheduling the trip when the reservation is placed reduces a substantial portion of the scheduling effort, as the confusing aspects of space and time are mitigated by the structure. Instead of seating potentially hundreds of trip requests to runs every day, the scheduler focuses on adding enhancements to improve the scheduled service.

To make a clear and quantifiable justification for implementing structured demand and help illustrate the concepts, a case study is presented using actual service data from a rural demand-response transportation system.

\section{Methodology}

Each methodology task first describes the methodology in general terms, then describes how the process applies to the case study site. This parallel structure enables replication of the methodology, while illustrating the structuring and enhancement process using a specific example. 


\section{Select a Site}

To apply all of the recommendations in this research, the study site must have certain characteristics that enable the scheduling process to be structured according to the research recommendations. These characteristics include:

- The service area has one, and only one, urban area.

- The urban area contains basic medical, retail, and employment centers.

- The urban area is located in or near the geographic center of the service area.

- Major roads provide direct access to the urban area from the outlying areas.

- The urban area is sufficiently dense to allow a vehicle to provide service to all major destinations within a reasonable time.

These characteristics are not present in all rural DRT systems. For systems that have different characteristics, the conceptual process of structuring demand and applying enhancements still applies, but the structure may need to be altered to achieve optimal results.

Case Study: Lenoir County, North Carolina, is the case study site used to ascertain the benefits of structured rural demand-response transportation. Lenoir County Transit (LCT), based in Kinston (population 21,677 [U.S. Census 2010]), is a rural community transportation system that serves the general public, medical, and human services trips for Lenoir County (population 59,495 [U.S. Census 2010]). Kinston is the capital of the county and contains basic health services, employment, and shopping destinations for Lenoir County. Few services exist within the county outside of the Kinston area. It is situated just north of the Neuse River, which divides Lenoir County in half while generally flowing west to east. The majority of Lenoir County's population and services are north of the river, with the notable exception of the community college across the river to the southeast. Kinston is served north/south by US 258 and east/ west by US 70 .

In Fiscal Year 2011 (July 2010-June 2011), LCT provided 107,019 passenger trips, traveled 812,372 service miles, and operated 18 vehicles (primarily lift vans) (NCDOT 2012). The average cost per service mile was $\$ 1.33$ in FY2011 (NCDOT 2012). LCT operates a deviated fixed route within Kinston with 16 roughly hourlong runs between $7 \mathrm{AM}$ and midnight. 


\section{Preparing Origin/Destination Data}

After selecting a site, trip data must be collected that includes origin and destination addresses, requested pickup/drop off times, and assigned runs. The addresses should include the physical street address and postal code, at a minimum. Optimally, the addresses will be collected as coordinate data to ensure accurate mapping.

The in-service area trips are split into two rows, with one line for the origin stop and one line for the destination stop. Trips that have an origin, destination, or both that are unable to be mapped due to incomplete or inaccurate addresses are removed. Out-of-service-area trips are removed, as these trips need to be served with a different structure than in-service area trips.

A time constraint code is added to each trip to make it easier to categorize trips based on the customer's needs. Demand-response trip data contains a pickup time and a drop off time, but only one of these times is defined by the customer, and thus, indicate the customer's need. Trips that originate at a customer's home are assigned a drop-off time constraint code, as it is likely that the customer is most concerned about when he/she arrives at the destination. Trips that terminate at a customer's home are deemed to be constrained by the pickup time.

The final step in preparing the data is to map the trip origins and destinations. The mapped origins/destinations show one point for the pickup and one for the dropoff. For this analysis, the data are mapped using a Geographic Information System (GIS). It is also possible to use Web-based mapping tools.

Case Study: LCT was able to provide one week's worth of data from August 2011. The week coincides with a state-mandated sampling period. The peak day (Wednesday) was selected for in-depth analysis. Roughly 10 percent of the trips were removed due to incomplete address information. There were a total of 344 usable trips for the Wednesday study period.

\section{Determine Actual Service}

It is essential to establish a baseline for comparison that enumerates the actual service provided by the rural DRT during the study period. The simplest and most consistent comparison using readily-available tools and data is to calculate the number of miles required to deliver the service. The number of passengers remains consistent between the actual service and the proposed service. Service hours are 
difficult to calculate, as assumptions must be made concerning the boarding and alighting times of passengers and groups of passengers.

It is possible to use the daily start and end odometers of the vehicles during the study period to calculate the miles only if 1 ) no trips were removed due to incomplete data and 2) no break/maintenance/fueling miles were incurred. If these situations exist, the most equitable method of calculating miles is to use GIS or an online mapping service to determine network miles using the pickup and drop-off times for each stop. The actual miles may not equate to service miles, but it does provide a level field for comparison.

In addition to the miles required to deliver the service, the peak and total number of vehicles used to deliver the service must be determined.

Case Study: For LCT data, it is not possible to use the daily start and end odometers to calculate miles because some incomplete addresses were removed. Instead, the miles are calculated by mapping every stop for every run in online mapping software. The stops were ordered according to how LCT performed them. It was not possible to know the exact path that the drivers used to travel between the stops, nor whether the vehicles returned to the depot during long stretches of inactivity. For these reasons, it is believed that the total miles of actual service is a conservative estimate. The actual service characteristics are displayed in Table 1.

\section{Table 1. Actual Service Characteristics for Lenoir County Transportation}

\begin{tabular}{lr}
\hline Vehicles & 17 \\
Peak Vehicles & 17 \\
Deviated Fixed-Route Trips & 59 \\
Demand-Response Trips & 285 \\
Total Miles & 2,742 \\
\hline
\end{tabular}

\section{Develop a Geographic Service Structure}

Creating service zones is the first step in structuring rural DRT service, with the central zone being the first zone to establish. The central zone should be as small as possible, yet contain all or almost all the potential destinations in the service area. A properly-defined central zone is essential to simplifying the scheduling process, as almost all trips from outer zones will terminate in the central zone for rural DRT systems with the previously-defined geographic characteristics. 
Next, outer zone boundaries are developed based on natural breaks in the transportation network, such as rivers, ridges, and undeveloped areas. These zones should be centered on the primary road network used to access the central zone. Roads should not be used as zone boundaries, as this may result, for example, in one vehicle serving the east side of the road and another vehicle serving the west side. Splitting neighborhoods, small communities, and other areas with distinct identities should be avoided where possible.

Outer zones should be sized to create manageable service areas. If a service area is large, there may be two or more layers of concentric outer zones, resembling a dart board. For smaller service areas, outer zones may span from the central zone to the edge of the service area, exhibiting a pie shape. Whatever the configuration of the outer zones may be, it is essential that these zones be easily understandable to both the schedulers and the customers. It is also essential that almost all of the trips originating in the outer zones terminate in the central zone.

Case Study: The central zone for LCT contains the city of Kinston and a small section southeast of Kinston to incorporate the local community college. Ninety-five percent of the trips in the LCT service area originate or terminate in the central zone. The outer zones are pie-shaped and named according to ordinal directions. Figure 1 shows the zones and the origin and destination stops for the selected study day. There are eight outer zones. The zone directly to the south of the central zone (indicated by hatching) warrants special consideration, as three other zones pass through it to access the central zone. As it is not possible to efficiently distribute the land area in this zone to the three outer zones, trips originating/terminating in this pass-through zone will be served by the zone runs that pass through it (zones W, SW, and SE).

\section{Structure Service}

Once the geographic service structure is in place, the daily scheduling problem becomes easier to solve, as the trips are categorized in groups based on time and space. The scheduling problem then becomes a tabular problem instead of a space and time problem. Trip reservations are scheduled to the appropriate zone run as the reservations are booked. In addition to recording the reservation details, the booking agent will update the scheduling table to reflect the number of trips for each run. 


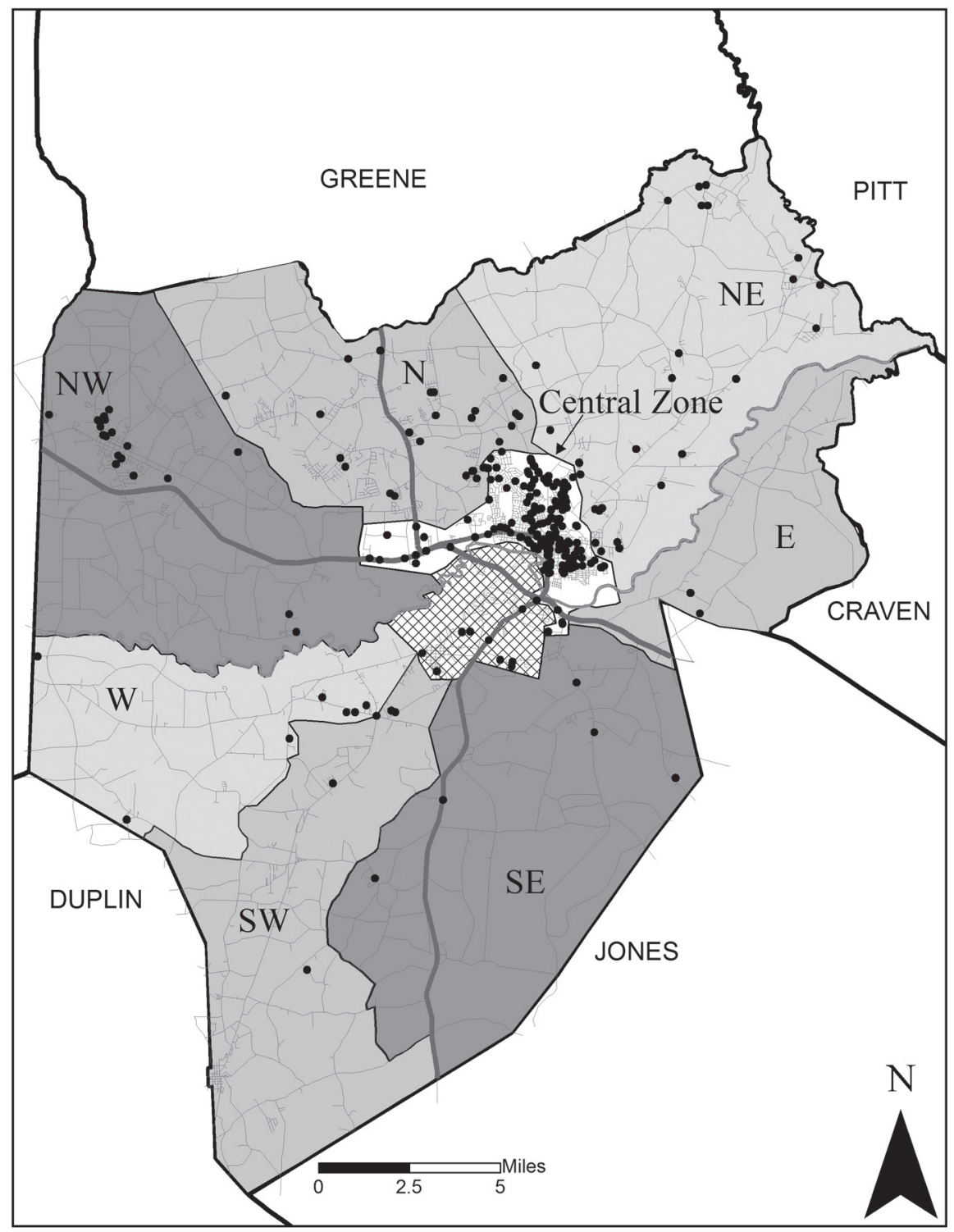

Figure 1. Zone-Based Scheduling Structure for Lenoir County 
Before daily service begins, the scheduler must first look over the table to see if there are any issues with vehicle capacity and add runs if necessary. The scheduler then adds the short-term enhancements of eliminating unnecessary deadhead (empty or unloaded runs) and consolidating runs where possible. Finally, the scheduler must determine the appropriate order to serve the trips within the zone-based runs.

Will-call trips are a common cause of inefficient service delivery. The structured demand makes it simple to schedule will-call trips. When the request is called in, the staff person can look at the scheduling matrix to determine the next run available to serve the trip request and assign the trip to that run. The end result of a scheduling structure will be a more efficient schedule and an easier scheduling process.

\section{Develop a Scheduling Table}

To begin structuring the service and creating a scheduling table, a service time period that meets the needs of the customers must first be determined. Each zone should be eligible to be served by inbound and outbound runs once per time period. For example, a run may originate in each outer zone every hour, and a run may terminate in each outer zone every hour (originating in the central zone). This example structure means that there may be two vehicles in each zone at every time period (one inbound, one outbound). Trips may also originate and terminate within the central zone every time period.

It is necessary to develop rules to assign trips to a zone-based run structure, as the customer's stated demand for a service time will not initially fit neatly into the zone structure. For example, a trip that is desired to be picked up in an outer zone and dropped off in the central zone at 9:30AM needs to be assigned to either the runs that originate in the outer zone at 8AM or 9AM. Because the customer most likely needs to be at the destination by 9:30AM, the trip should be assigned to the 8AM run, which drops off all passengers in the central zone by 9AM.

After developing the assignment rules, each trip is categorized by time period into the zone-based run structure. The structured service may then be displayed in a table with the columns displaying runs and the rows displaying the time period, and the total trip requests in the associated run cells (see the case study section for an example). Runs that originate in the outer zones are inbound, while runs that originate in the central zone are outbound. 
Trips that originate and terminate in the central zone and trips that originate and terminate outside of the central zone are assigned to their own runs. If fixed-route or deviated fixed service exists, all trips should remain assigned to these services. The remaining trips are assigned to sweeper vehicles that stay within the central zone. Rural sweeper vehicles are used to capture trips that do not originate/terminate in the central zone.

Additional runs are added where demand exceeds capacity. For example, more than one central zone sweeper may be required to serve the trip demand. A simple method of determining how many runs are required to serve the demand is to establish a maximum trip count that can be adequately served by the run within the constraints. Each zone may have unique maximum trip counts depending on the constraints.

Next, a starting and ending point should be established for each run. Each run that originates in the outer zone must have an accompanying run the previous time period that terminates in the outer zone. In addition, each run that terminates in the outer zone must have an accompanying run the following time period to move the vehicle back to the central zone. Sometimes starting and ending points must be reached with deadhead runs.

After categorizing the trips into the structured service, GIS or web-based mapping is used to calculate the average number of miles required to serve each zone. To be conservative, it is assumed that each outbound run originates at the depot and each inbound run terminates at the depot.

Finally, the number of runs for the new service structure is multiplied by the average number of miles to determine the structured miles. This number will serve as the baseline for comparison with the actual service and the enhancements. In addition, the total runs for each time period are calculated, which determines the number of vehicles required per time period.

At this point, a scheduling table has been established based on the customer's stated demand. The next steps are to add enhancements to improve efficiency.

Case Study: Trips for LCT are assigned to runs based on a time period of one hour. The scheduling goal for rural zones was to have roughly four trips per run to allow for all trips to be served within the one hour timeframe. Urban zones were allowed six trips per run. Deadhead runs with zero trips are established to move the vehicles to/from the appropriate zones. To provide adequate service, LCT requires up to four central zone sweepers and two rural sweepers in addition to the deviated fixed route. LCT requires a 
maximum of two vehicles per rural zone, one inbound and one outbound. There is one instance (NW in at 7 AM) where six trips are served by one rural run. As the preceding outbound run is empty, it should not be an issue for this run to serve 6 trips. The total structured miles for LCT is essentially the same as the total actual miles (2,742 actual miles; 2,735 structured miles), and total and peak number of vehicles is identical (17).

Table 2. Scheduling Table Based on Stated Demand

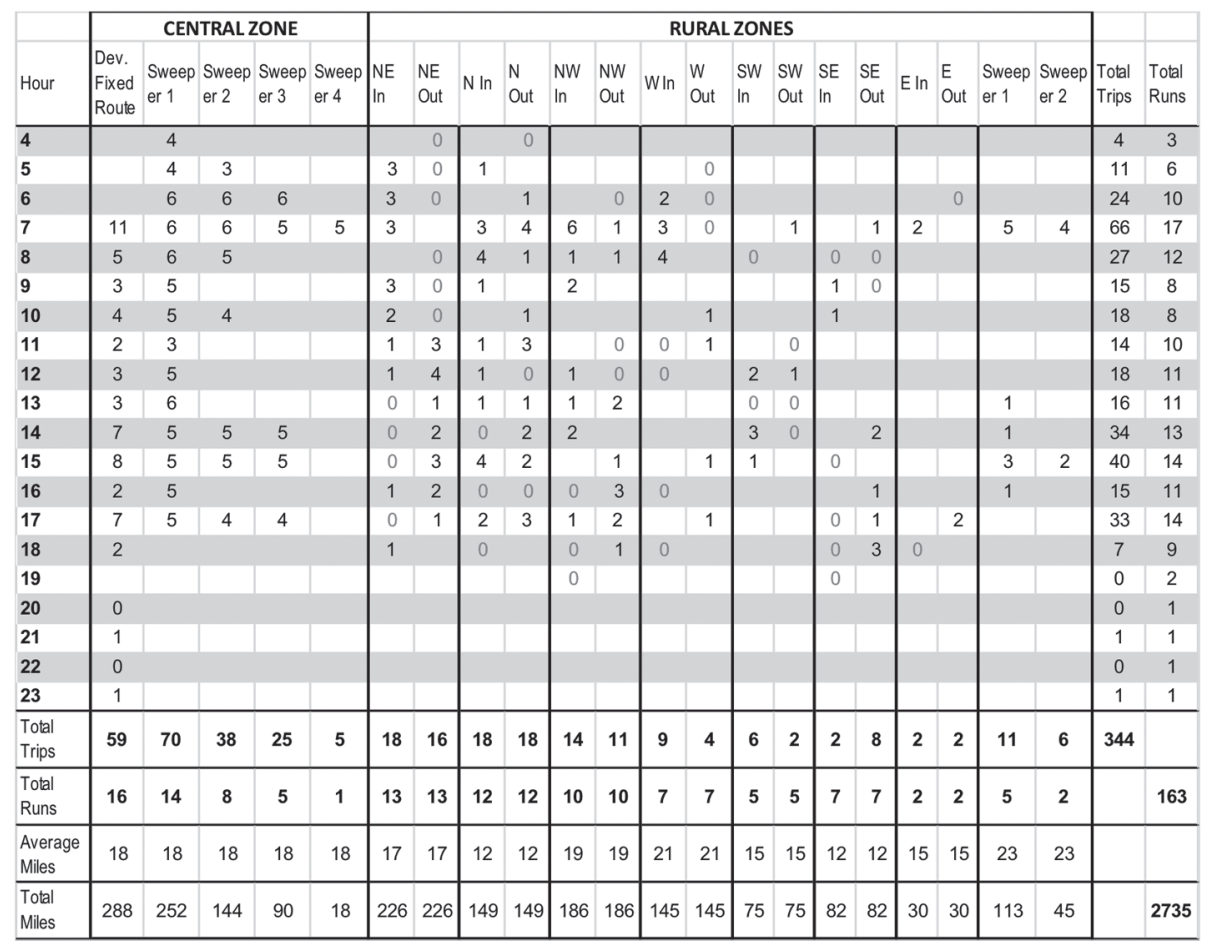

\section{Add Enhancements}

Once the scheduling process is structured, it is relatively simple to add performance enhancements. The structure is essential, however, as understanding the disparate pickup and drop-off demands stated by the customers is difficult for the scheduler to process as trip requests increase.

There are two categories of enhancements, long-term and daily. Long-term enhancements include structuring demand and outstationing vehicles. Daily enhancements include eliminating unnecessary deadhead runs and consolidating runs with low trip counts. 
Demand for trips outside of the structured service times and locations should be tracked to assist with updating the scheduling structure in the future. Updates to the structure should be considered on a regular basis to ensure that the transit system is responding to the needs of its customers.

In this research, enhancements were applied only to rural zones that serve the central city. Central city runs and rural sweeper runs must be dealt with using different scheduling processes than what is proposed in this research. For example, increasing fixed-route service or redesigning the service may reduce the number of central city sweeper runs, while implementing a hub-and-spoke service design or brokerage may eliminate the need for rural sweeper vehicles. However, these scheduling and planning methods are outside of the scope of this research.

\section{Long-Term Enhancement 1: Structured Demand}

Instead of adapting to the stated demand of the customers (who have no framework within which to place their demands), the rural DRT should structure the demand using historical data. By categorizing trips into geographic service areas and times, it is possible to determine the demand for service within a zone at each time period. Rural DRTs should determine service demand within the structured zone-based framework and establish a structured demand-based service where runs with little or no ridership are eliminated.

It is preferable to have the same structured demand for each weekday. First, a scheduling table for each weekday should be developed. Next, runs with low demand should be eliminated. If service fluctuates greatly between weekdays, it may be necessary to develop scheduling tables for individual days of the week.

Case Study: By eliminating runs with little or no demand, 30 runs were removed from service, resulting in a savings of 452 miles (25\%). Six runs were eliminated for the NE zone, 6 for the N zone, 10 for the NW zone, 2 for the $W$ zone, 2 for the SW zone, and 4 for the SE zone. To accomplish this structure, only 27 trips were moved from their original demanded time. Trips initially assigned to the eliminated runs were moved to the preceding run before noon and the following run after noon to account for trip time constraints. Central zone runs and rural sweeper runs are not affected by the structured demand enhancement. The final structured service, after unproductive runs were removed, is shown in Table 3. This structure became the baseline for determining the impact of the enhancements outlined in the following section. The proposed structured demand results in a savings of 453 miles and $\$ 602$ per day, when compared to the actual miles. 
Table 3. Scheduling Table Based on Structured Service

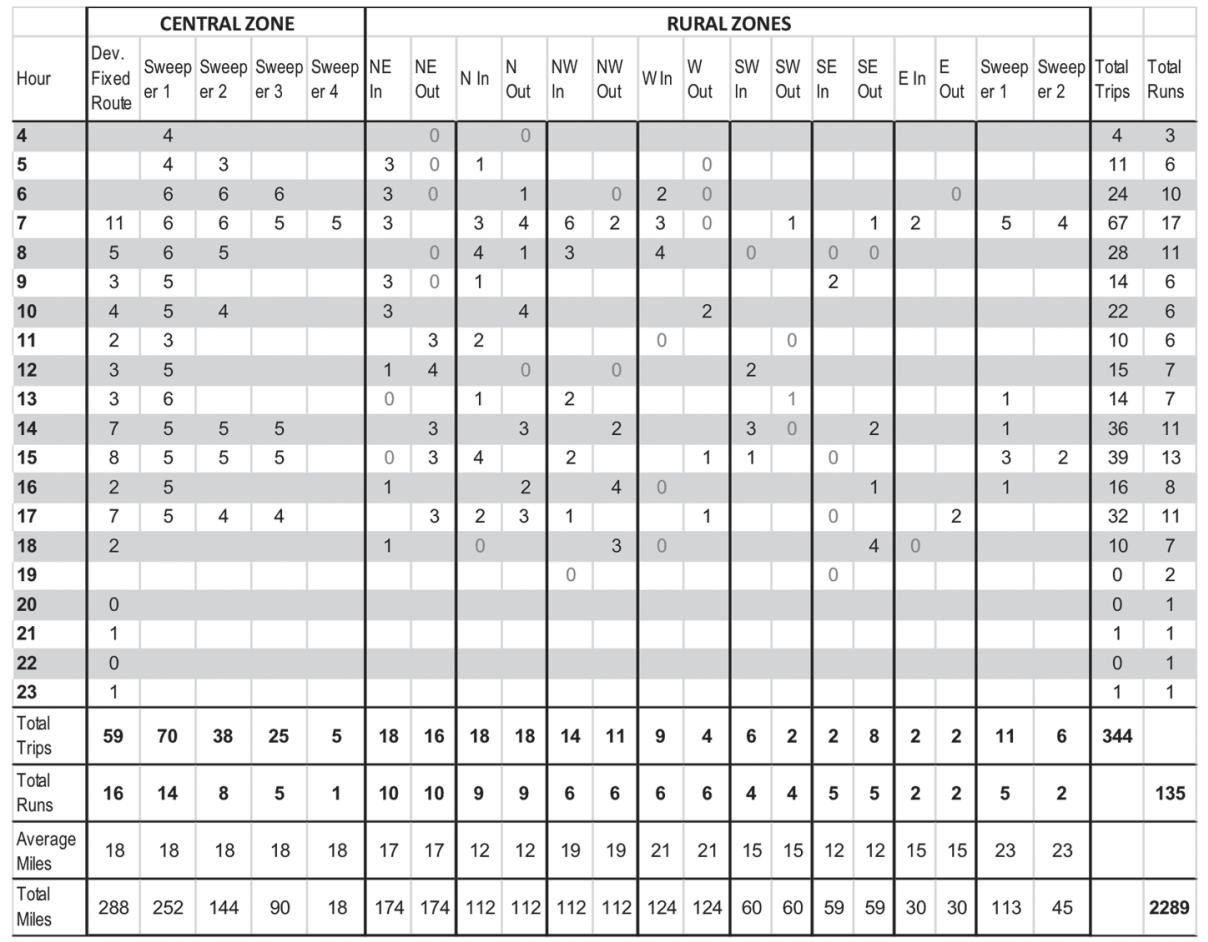

\section{Long-Term Enhancement 2: Outstationing Vehicles}

Outstationing vehicles can be more easily accomplished in a structured demand environment because it is known when and where each run will begin and end. In pure demand-response service, it is difficult to outstation vehicles because the service changes on a daily basis. Depending on the structure, oustationing vehicles in safe, secure locations may result in decreased deadhead miles from the depot to the start of a run originating in a rural zone and vice versa. Outstationing requires that a morning outstationed run be accompanied by an afternoon run that terminates at the outstation site.

Case Study: Table 4 shows the scheduling table with both long-term enhancements, which becomes the baseline scheduling table for adding daily enhancements. Outstationing seven vehicles results in a 16 percent reduction in miles (233 miles) from the structured service baseline. Zones NE, N, NW, SE, and E each have one outstationed vehicle. Zone $W$ has two outstationed vehicles. Zone SW has no outstationed vehicles. Adding outstationing to the structured service results in an estimated daily savings of $\$ 310$ (233 miles $x$ 
$\$ 1.33$ cost per mile) from the structured service scheduling table. Together, the long-term enhancements of outstationing and structuring the service resulted in an estimated daily savings of $\$ 912$ (686 miles $x \$ 1.33$ cost per mile), when compared to the actual service.

\section{Table 4. Scheduling Table Based on Long-Term Enhancements}

\begin{tabular}{|c|c|c|c|c|c|c|c|c|c|c|c|c|c|c|c|c|c|c|c|c|c|c|c|}
\hline \multirow[b]{2}{*}{ Hour } & \multicolumn{5}{|c|}{ CENTRAL ZONE } & \multicolumn{16}{|c|}{ RURALZONES } & \multirow[b]{2}{*}{$\begin{array}{l}\text { Total } \\
\text { Trips }\end{array}$} & \multirow[b]{2}{*}{$\begin{array}{l}\text { Total } \\
\text { Runs }\end{array}$} \\
\hline & $\begin{array}{l}\text { Dev. } \\
\text { Fixed } \\
\text { Route }\end{array}$ & $\begin{array}{l}\text { Sweep } \\
\text { er } 1\end{array}$ & $\begin{array}{l}\text { Sweep } \\
\text { er } 2\end{array}$ & $\begin{array}{l}\text { Sweep } \\
\text { er } 3\end{array}$ & $\begin{array}{l}\text { Sweep } \\
\text { er } 4\end{array}$ & $\begin{array}{l}\mathrm{NE} \\
\text { In }\end{array}$ & $\begin{array}{l}\mathrm{NE} \\
\text { Out }\end{array}$ & $N \ln$ & $\begin{array}{l}\mathrm{N} \\
\text { Out }\end{array}$ & $\begin{array}{l}\text { NW } \\
\text { In }\end{array}$ & $\begin{array}{l}\text { NW } \\
\text { Out }\end{array}$ & WIn & $\begin{array}{l}\text { W } \\
\text { Out }\end{array}$ & $\begin{array}{l}\text { SW } \\
\text { In }\end{array}$ & $\begin{array}{l}\text { SW } \\
\text { Out }\end{array}$ & $\begin{array}{l}\text { SE } \\
\text { In }\end{array}$ & $\begin{array}{l}\text { SE } \\
\text { Out }\end{array}$ & $E \ln$ & Eut & $\begin{array}{l}\text { Sweep } \\
\text { er } 1\end{array}$ & $\begin{array}{l}\text { Sweep } \\
\text { er } 2\end{array}$ & & \\
\hline 4 & & 4 & & & & & & & & & & & & & & & & & & & & 4 & 1 \\
\hline 5 & & 4 & 3 & & & 3 & 0 & 1 & & & & & & & & & & & & & & 11 & 5 \\
\hline 6 & & 6 & 6 & 6 & & 3 & 0 & & 1 & & & 2 & & & & & & & & & & 24 & 7 \\
\hline 7 & 11 & 6 & 6 & 5 & 5 & 3 & & 3 & 4 & 6 & 2 & 3 & 0 & & 1 & & 1 & 2 & & 5 & 4 & 67 & 17 \\
\hline 8 & 5 & 6 & 5 & & & & 0 & 4 & 1 & 3 & & 4 & & 0 & & 0 & & & & & & 28 & 10 \\
\hline 9 & 3 & 5 & & & & 3 & 0 & 1 & & & & & & & & 2 & & & & & & 14 & 6 \\
\hline 10 & 4 & 5 & 4 & & & 3 & & & 4 & & & & 2 & & & & & & & & & 22 & 6 \\
\hline 11 & 2 & 3 & & & & & 3 & 2 & & & & 0 & & & 0 & & & & & & & 10 & 6 \\
\hline 12 & 3 & 5 & & & & 1 & 4 & & 0 & & 0 & & & 2 & & & & & & & & 15 & 7 \\
\hline 13 & 3 & 6 & & & & 0 & & 1 & & 2 & & & & & 1 & & & & & 1 & & 14 & 7 \\
\hline 14 & 7 & 5 & 5 & 5 & & & 3 & & 3 & & 2 & & & 3 & 0 & & 2 & & & 1 & & 36 & 11 \\
\hline 15 & 8 & 5 & 5 & 5 & & & 3 & 4 & & 2 & & & 1 & 1 & & 0 & & & & 3 & 2 & 39 & 12 \\
\hline 16 & 2 & 5 & & & & 1 & & & 2 & & 4 & & & & & & 1 & & & 1 & & 16 & 7 \\
\hline 17 & 7 & 5 & 4 & 4 & & & 3 & 2 & 3 & 1 & & & 1 & & & 0 & & & 2 & & & 32 & 11 \\
\hline 18 & 2 & & & & & 1 & & & & & 3 & & & & & & 4 & & & & & 10 & 4 \\
\hline 19 & & & & & & & & & & & & & & & & & & & & & & 0 & 0 \\
\hline 20 & 0 & & & & & & & & & & & & & & & & & & & & & 0 & 1 \\
\hline 21 & 1 & & & & & & & & & & & & & & & & & & & & & 1 & 1 \\
\hline 22 & 0 & & & & & & & & & & & & & & & & & & & & & 0 & 1 \\
\hline 23 & 1 & & & & & & & & & & & & & & & & & & & & & 1 & 1 \\
\hline \begin{tabular}{|l|} 
Total \\
Trips \\
\end{tabular} & 59 & 70 & 38 & 25 & 5 & 18 & 16 & 18 & 18 & 14 & 11 & 9 & 4 & 6 & 2 & 2 & 8 & 2 & 2 & 11 & 6 & 344 & \\
\hline \begin{tabular}{|l|} 
Total \\
Runs \\
\end{tabular} & 16 & 14 & 8 & 5 & 1 & 9 & 9 & 8 & 8 & 5 & 5 & 4 & 4 & 4 & 4 & 4 & 4 & 1 & 1 & 5 & 2 & & 121 \\
\hline $\begin{array}{l}\text { Average } \\
\text { Miles } \\
\end{array}$ & 18 & 18 & 18 & 18 & 18 & 17 & 17 & 12 & 12 & 19 & 19 & 21 & 21 & 15 & 15 & 12 & 12 & 15 & 15 & 23 & 23 & & \\
\hline \begin{tabular}{|l|} 
Total \\
Miles
\end{tabular} & 288 & 252 & 144 & 90 & 18 & 157 & 157 & 99 & 99 & 93 & 93 & 83 & 83 & 60 & 60 & 47 & 47 & 15 & 15 & 113 & 45 & & 2056 \\
\hline
\end{tabular}

\section{Daily Enhancement: Consolidate Runs}

Once the long-term enhancements are in place, the scheduler must focus on improving the daily schedule by consolidating runs. There are two steps to consolidate runs. The first step is to eliminate unnecessary deadhead runs, which occur when one vehicle deadheads empty in one direction while another deadheads empty in the other direction. The next step is to combine runs in adjacent zones that have low trip counts. Runs must be consolidated in pairs, as there is no benefit to consolidating two outbound runs only to have to add an additional deadhead run to account for the following inbound runs.

Consolidating runs will likely result in mileage increases, as a vehicle may need to travel across zones. The increased miles must be considered by the scheduler when determining the effectiveness of run consolidation. 
Case Study: It is assumed for LCT that every consolidated run results in an additional 10 miles of travel for the unconsolidated run to account for travel between zones. In addition, runs may only be consolidated if they are in the same or adjacent zones. With these rules in place, four runs are consolidated to eliminate unnecessary deadhead runs and two are consolidated due to low demand. The deadhead runs that were eliminated are N Outbound at noon, NE Inbound at 1 PM, SW Outbound at 2 $P M$, and SE Inbound at 3 PM. The low demand runs that were consolidated are $W$ and SW Outbound at 7 AM; and W and SW Inbound at 8 AM. Table 5 shows the scheduling table after applying the daily enhancements. The consolidation of 6 runs resulted in a total savings of 62 miles from the long-term enhancement baseline after 60 additional miles are added to account for travel between the zones. The total savings of structuring demand and adding enhancements is 748 miles (a 27\% reduction from actual miles) and an estimated daily savings of \$995. In addition, the simplified scheduling process resulted in a reduction of staff time and effort.

\section{Table 5. Scheduling Table with Daily Enhancements}

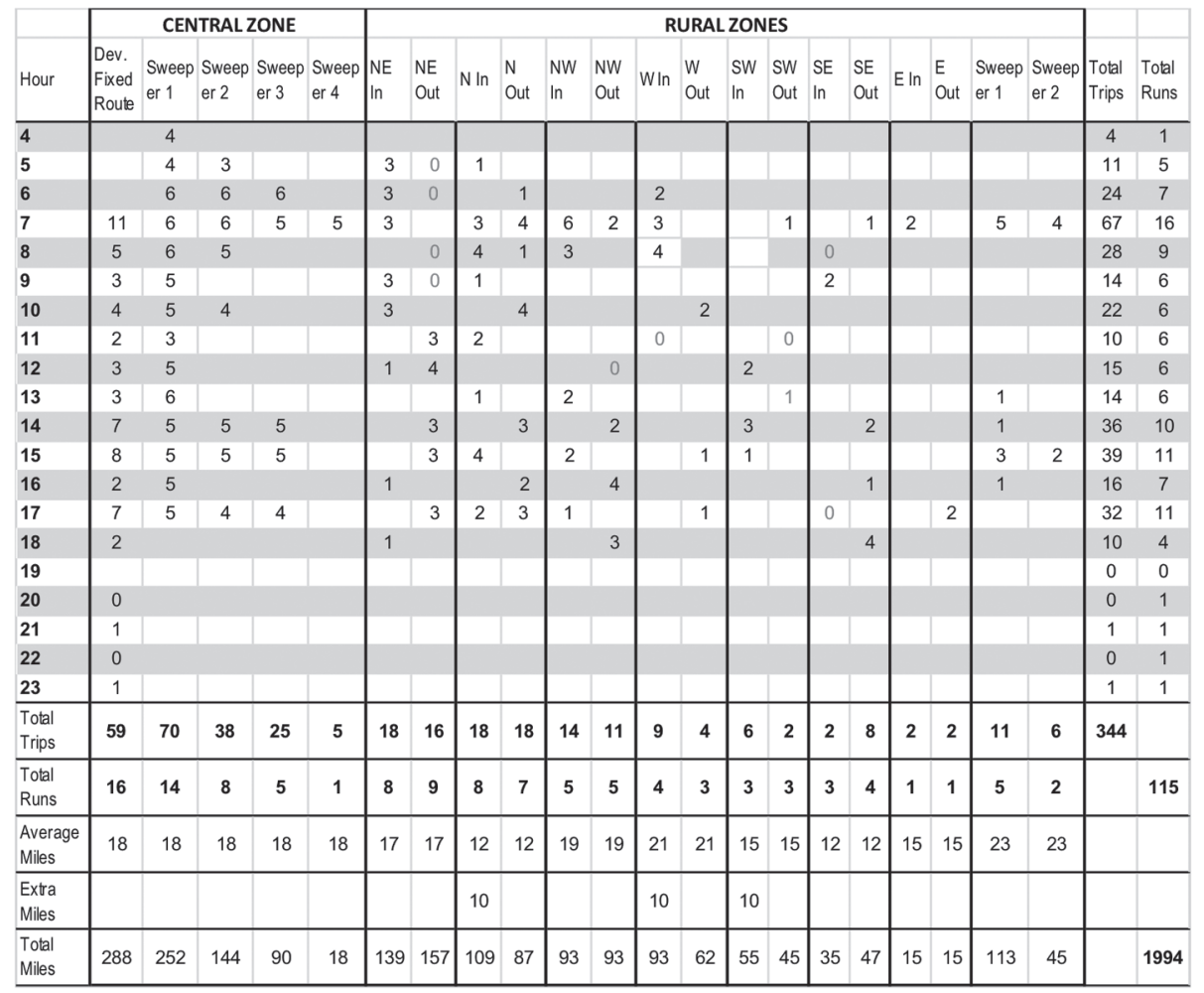




\section{Conclusions}

Scheduling rural demand-response trips is difficult, time-consuming, and often results in inefficient service delivery and inefficient use of resources. As rural demand-response service increases, providers often look to increase vehicle and scheduling capacity to handle the increased demand and complexity. Instead of adding resources, capacity can be expanded and performance can be increased by applying a well-planned scheduling structure.

This research explains how to establish structured rural demand-response transportation service and enumerates its benefits through a case study consisting of actual service data for a rural DRT system. Once a structure is in place, the trips are assigned to runs as they are booked. Grouping trips according to a structure based on historical demand simplifies the scheduling process by transforming it to a tabular exercise instead of a space-and-time exercise. The scheduler need not be concerned with seating trips on runs and can instead focus on improving the efficiency of the daily service.

Applying the recommendations cited in this research to the case study site results in a daily savings of 748 miles (a $27 \%$ reduction in miles) and $\$ 995$. The same number of trips is served, and the service structure is based on the customer's stated demand for service.

\section{References}

NCDOT Public Transportation Division. 2012. FY11 Operating Statistics (unpublished).

United States Census. 2010. Census Quickfacts 2010. Accessed April 24, 2012. http://quickfacts.census.gov/qfd/states/37/37107.html.

\section{About the Authors}

KAI MONAST (kcmonast@ncsu.edu) is a Senior Research Associate with the Institute for Transportation Research and Education at North Carolina State University and a member of the Transportation Research Board Paratransit Committee. He has a master's degree in Regional Planning and a bachelor's degree in Geography. His primary focus is on improving the efficiency of rural demand-response transportation service delivery. He assists North Carolina rural demand-response transportation 
systems with implementing technology, develops and provides training, and collects and analyzes rural DRT performance statistics.

JOSHUA WORTHY (jdworthy@ncsu.edu) is a Research Associate at the Institute for Transportation Research and Education at North Carolina State University. He has a bachelor's degree in Environmental Science. He collects and analyzes North Carolina rural demand-response transportation data and provides technical assistance to transit systems with a goal of improving performance. 